\title{
Arteritis de Takayasu en Lactante. Caso Clínico
}

\author{
CAROLINA ABARZÚA C. ${ }^{1}$, SARA SOTO M. ${ }^{2}$, GLORIA GONZÁLEZ G. ${ }^{3}$, FRANCISCO SAITÚA D. ${ }^{3}$, \\ ADOLFO DOLZ A. ${ }^{3}$, MERCEDES GUEVARA V. ${ }^{4}$, CARLOS ACUÑA A. ${ }^{2}$ \\ 1. Médico cirujano, Becado de Pediatría, Hospital Luis Calvo Mackenna, Facultad de Medicina, Universidad de Chile. \\ 2. Pediatra intensivista, Unidad de Cuidados Intensivos, Hospital Luis Calvo Mackenna. \\ 3. Cirujano infantil, Servicio Cirugía, Hospital Luis Calvo Mackenna. \\ 4. Pediatra, Unidad de Lactantes, Hospital Luis Calvo Mackenna.
}

\begin{abstract}
\section{Takayasu arteritis in childhood, a clinical case}

Background: Takayasu arteritis (TA) is a large vessel systemic vasculitis, affecting the aorta and its main branches, leading to stenosis, thrombosis, and aneurysm formation. Incidence of 2.6 cases per million per year has been reported. TA is rare condition in children. Steroids are the main therapy for active disease, however, additional immunosuppressive agents are required in 50\% of the patients. Objective: To report a case of TA in a young infant, presenting with intestinal necrosis. Case Report: 12 month-old male diagnosed with TA at 6 month-old, treated with second line immunosupressors. He presented with 3 days of abdominal pain, images showed aneurysm and thrombosis of the superior mesenteric artery. Small bowel ischemia was confirmed. The patient underwent multiple surgical interventions with bowel resection, leaving $30 \mathrm{~cm}$ of jejunum and $10 \mathrm{~cm}$ of terminal ileum. Anticoagulant therapy was started immediately. Intestinal continuity was restored 6 weeks later, patients was discharge home 3 months after surgery in full enteral intake, anticoagulant therapy, and maintains immunosupressor therapy. Discussion: TA in young infants is uncommon, and they can present atypical clinical manifestations. These patients are a real challenge, requiring multidisciplinary care to avoid further morbidities related to the vasculitis.
\end{abstract}

(Key words: Vasculitis, Takayasu Arteritis, mesenteric thrombosis, steroids, immunosuppresor).

Rev Chil Pediatr 2011; 82 (1): 56-62

\section{RESUMEN}

Introducción: La Arteritis de Takayasu (AT) es una vasculitis de grandes vasos, que afecta a la aorta y sus ramas principales, llevando a estenosis, trombosis, y formación de aneurismas. Se ha reportado una incidencia de 2.6 casos por millón por año, siendo de baja incidencia en niños. La terapia de elección son los corticoides, seguidos de inmunosupresores en casos resistentes. Objetivo: Reportar el caso de un lactante con AT severa,

Trabajo recibido el 10 de enero de 2011, devuelto para corregir el 26 de enero de 2011, segunda versión el 05 defebrero de 2011, aceptado para publicación el 20 de febrero de 2011.

Correspondencia a:

Dra. Carolina Abarzúa C.

E-mail: cabarzuac@gmail.com 
que cursó con necrosis intestinal. Caso Clínico: Varón, 12 meses de edad, diagnóstico de AT a los 6 meses, en tratamiento de segunda línea con inmunosupresores. Presentó cuadro de 3 días de evolución de dolor abdominal, imágenes mostraron trombosis de arteria mesénterica superior aneurismática, desarrollando isquemia intestinal. Fue sometido a múltiples intervenciones quirúrgicas con resección intestinal, con $30 \mathrm{~cm}$ de yeyuno y $10 \mathrm{~cm}$ de ileon terminal remanente. Inició precozmente tratamiento anticoagulante. La reconstitución de tránsito intestinal fue 6 semanas más tarde, paciente fue dado de alta 3 meses después alimentándose completamente por vía enteral, con tratamiento anticoagulante e inmunosupresor ambulatorio. Conclusión: La AT en lactantes es rara, y las manifestaciones clínicas pueden ser atípicas. Estos pacientes son un desafío para los equipos médicos, y requirien un manejo multidisciplinario para evitar complicaciones mayores.

(Palabras clave: Vasculitis, arteritis de Takayasu, trombosis mesénterica, corticoides, inmunosupresores).

Rev Chil Pediatr 2011; 82 (1): 56-62

\section{Introducción}

La Arteritis de Takayasu (AT) corresponde una vasculitis granulomatosa idiopática crónica, que afecta a la aorta y a sus ramas principales ${ }^{1}$.

En pediatría las vasculitis son una entidad poco frecuente, la AT ocupa el tercer lugar en frecuencia ${ }^{2}$, tras el purpura de SchonleinHenoch y la enfermedad de Kawasaki.

La incidencia de AT se estima en 2,6 casos por millón de personas por año ${ }^{3}$. Afecta con mayor frecuencia a mujeres, entre la segunda y tercera década de la vida. En niños se describe una edad media de inicio de 11,4 años, en su mayoría niñas $(75 \%)^{4}$.

Desde el punto de vista histológico se observa un infiltrado linfomonocitario y, ocasionalmente células gigantes con presencia de granulomas, afectando principalmente a la adventicia, pero progresa hacia la luz arterial en forma de panarteritis ${ }^{5}$. El proceso inflamatorio de la AT provoca trombosis en las arterias afectadas y aparición de estenosis, dilatación y aneurismas. Los hallazgos clínicos más comunes se derivan de la isquemia de miembros o los órganos afectados.

La Academia Americana de Reumatología elaboró criterios diagnósticos para AT (Tabla 1). Pero dichos criterios no se presentan en etapas tempranas de enfermedad, donde se manifiestan síntomas de enfermedades sistémicas como artralgias, anorexia, fiebre, astenia, mialgias. En niños series clínicas describen hipertensión arterial sistémica como principal hallazgo, seguido por fiebre, palpitaciones, vómitos, artralgias, pérdida de peso, claudicación, dolor abdominal y torácico, sumado a criterios de laboratorio como velocidad de sedimentación globular (VSG) elevada y cardiomegalia ${ }^{4}$. Últimamente progresos en técnicas de imagen permiten diagnosticar con exactitud y contribuyen al seguimiento de este tipo de pacientes, destacando la resonancia nuclear magnética (RNM), angioresonancia magnética (AngioRNM), tomografía computerizada (TC), Angiotomografía computerizada (AngioTAC), ecografía doppler y tomografía de emisión de positrones (PET). Considerando la dificultad en el diagnóstico en etapas iniciales, surgen Criterios de Enfermedad Activa (tabla $2)^{6}$, la aparición o el empeoramiento de 2 o más rasgos o características de la Arteritis de Takayasu definen la enfermedad activa ${ }^{5,6}$.

En cuanto al tratamiento los corticoides son la terapia de primera línea, la mitad de los pacientes no responden o presentan reacciones adversas y requieren drogas citotóxicas para disminuir los síntomas asociados a la inflamación sistémica en la fase activa de la enfermedad ${ }^{7}$. Últimamente se describe el uso de e inhibidores del TNF $\alpha$ (Infliximab) como tratamiento ante casos refractarios ${ }^{8}$. Procedimientos quirúrgicos y angioplastía transluminal percutánea han sido descritas también, para tratar complicaciones del tipo esteno-oclusivas o lesiones aneurismáticas en etapa crónica².

La hipertensión arterial (HTA), debida principalmente a la afectación de aorta abdominal y arterias renales, lidera la lista de complicaciones en este tipo de pacientes ${ }^{9,10}$. Seguidas por la isquemia renal y mesentérica, para lo cual 
Tabla 1 Criterios diagnósticos de A. de Takayasu de acuerdo al Colegio Americano de Reumatología

\begin{tabular}{|l|}
\hline Comienzo antes de los 40 años \\
Claudicación de una extremidad \\
Disminución del pulso braquial \\
Diferencia de presión sistólica mayor a $10 \mathrm{mmHg}$ entre los brazos \\
Soplos sobre las arterias subclavias y/o aorta \\
Evidencias radiológicas de estenosis u oclusión de la arteria aorta y de sus ramas principales \\
Para el diagnóstico requiere al menos 3 de los 6 criterios
\end{tabular}

existen junto con el tratamiento anticoagulante y trombolítico sistémico habitual, tratamientos trombolíticos locales, tras cateterizar arteria afectada mediante radiología intervencional, este tipo de intervenciones presta mucho más seguridad que la terapia anticoagulante o trombolítica sistémica.

El objetivo es presentar el caso de un lactante de 12 meses en que a los 6 meses de vida se establece el diagnóstico de arteritis de takayasu, complicado con un cuadro de isquemia mesentérica que requirió resolución quirúrgica.

\section{Caso clínico}

Lactante varón, 12 meses de edad, sin antecedentes mórbidos, derivado a los 5 meses de edad al policlínico de cardiología del Hospital Luis Calvo Mackenna por circulación colateral en tórax y abdomen, se solicitó estudio ecográfico que mostró arteria intercostal prominente que se comunicaba con arteria mamaria izquierda aneurismática. Se exploró también aorta abdominal encontrándose dilatación aneurismática de arteria mesentérica superior que cuadriplica el diámetro de la aorta vecina. Dos semanas más tarde mediante nueva ecografía doppler se constató compromiso aneurismático de iliacas.

Evolucionó con HTA de díficil manejo, requiriendo terapia antihipertensiva combinada. Además se pesquisó diferencia de presión arterial mayor de $10 \mathrm{~mm} / \mathrm{Hg}$ entre ambas extremidades inferiores. Se objetivó una velocidad de eritrosedimentación (VSG) elevada,
HTA sistémica, asimetría en presión arterial de miembros inferiores, por lo cual se planteó diagnóstico clínico de Arteritis de Takayasu activa, basándose en criterios de enfermedad activa (Tabla 2), y se inició tratamiento con prednisona en forma ambulatoria.

Un mes más tarde, se realizó AngioTAC (figura 1) que informó aneurismas viscerales múltiples, en tronco celíaco y sus ramas, arterias renales, mesentéricas, hipogástricas, glúteas y ramas femorales profundas. Aneurismas también de arteria mamaria interna y ramas axilares izquierdas. Tras dicho resultado se decidió iniciar tratamiento con metotrexato por extensión de compromiso vascular en corto plazo. Al mes de tratamiento con metotrexato + corticoides se realizó ecografía de control que mostró aumento de diámetro de aneurisma mesentérico (14-17 mm), por lo que se decidió modificar tratamiento inmunosupresor por ciclofosfamida mensual. Se repitió ecografía un mes más tarde, sin cambios en diámetros arteriales, pero arterias iliacas y glúteas se observaron trombosadas. Se indicó tratamiento con ácido acetil salicílico en forma ambulatoria.

Al año de edad se hospitalizó por cuadro de abdomen agudo, ecotomografía abdominal y TAC de abdomen fueron sugerentes de obstrucción intestinal, el paciente fue intervenido objetivándose asa isquémica a nivel de íleon, se resecó $25 \mathrm{~cm}$ de ileon y apéndice cecal (figura 2).

Considerando riesgo de repetir fenómeno embólico se inició tratamiento anticoagulante con fragmin (dalteheparina).

Evoluciona en forma tórpida, por lo que se 
Tabla 2. Criterios de Enfermedad activa en pacientes con Arteritis de Takayasu

1.- Síntomas sistémicos, como fiebre o muscoloesqueléticos (descartando otras causas)
2.- Elevación de VSG
3.- Síntomas de isquemia vascular o inflamación, como claudicación, disminución o ausencia de pulso, soplo audible, dolor vascular,
asimetría de PA de miembros superiores o inferiores
4.- Hallazgos típicos en angiografía
La aparición (brote) o empeoramiento de dos o más criterios indican enfermedad activa

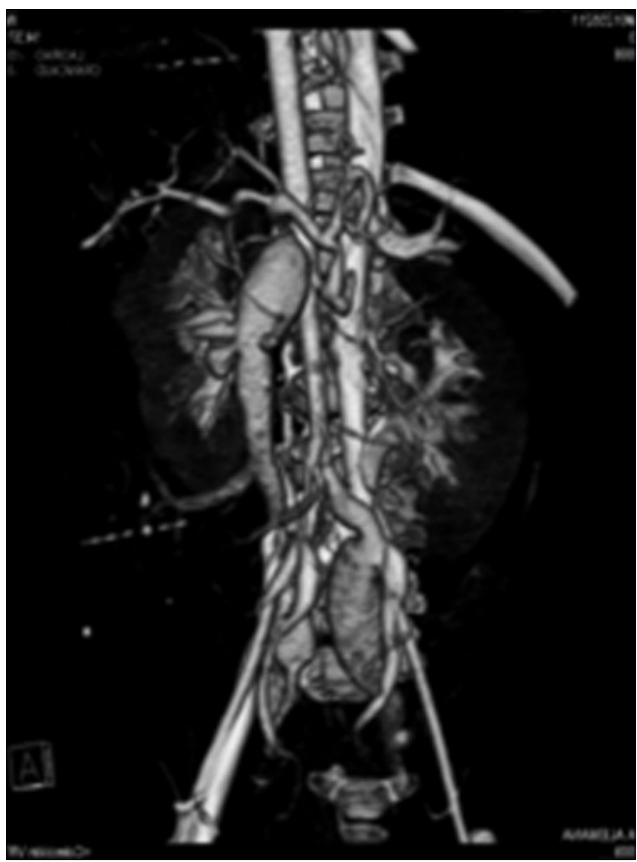

Figura 1. Reconstitución AngioTAC donde se observan aneurismas viscerales múltiples, en tronco celíaco y sus ramas, arterias renales, mesentéricas, hipogástricas, glúteas y ramas femorales profundas. Aneurismas también de arteria mamaria interna y ramas axilares izquierdas.

decidió reexploración quirúrgica a los 7 días, constatándose zona necrótica de aproximadamente $12 \mathrm{~cm}$ proximal a anastomosis, con 3 zonas perforadas, además de un trombo en el mesenterio. Se resecaron aproximadamente $20 \mathrm{~cm}$ de intestino de zona necrótica descrita, quedando $80 \mathrm{~cm}$ de intestino de dudosa vitalidad. Se realizaron 2 ostomias.

Dada la alta probabilidad de progresión de isquemia intestinal por trombos en arteria mesentérica y ante la imposibilidad de realizar tratamiento trombolítico local en nuestro cen-

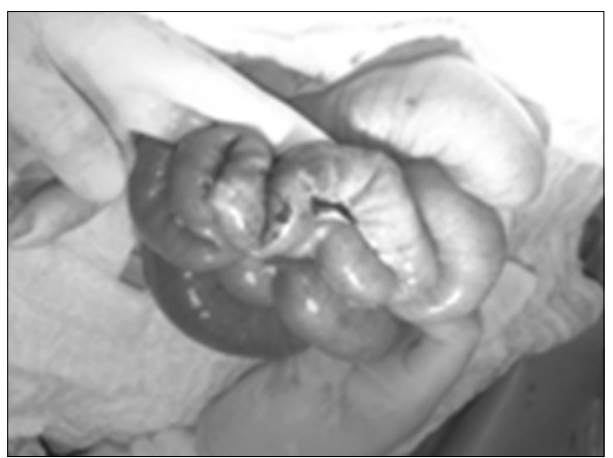

Figura 2 Figura de primera intervención donde se objetiva asa isquémica a nivel de íleon.

tro, se decidió iniciar tratamiento trombolítico sistémico con estreptoquinasa, pero se suspendió a las 24 horas de infusión por complicación hemorrágica.

Al día siguiente se apreció ostomía proximal de aspecto desvitalizada, decidiéndose realizar nueva AngioTAC. Dicho examen (figura 3) reveló trombosis de segmento distal de arteria mesentérica superior aneurismática junto con signos de isquemia de intestino delgado con posible perforación de un asa y colección intraperitoneal. Se realizó nueva intervención, resecando $30 \mathrm{~cm}$ de íleon, se realizó yeyunostomía proximal, quedando como remanente 30 $\mathrm{cm}$ de yeyuno más $10 \mathrm{~cm}$ de ileón, conservando válvula ileocecal.

Se reinició anticoagulación inicialmente con heparina pero 4 días más tarde se cambia a dalteheparina.

En consideración de que AngioTAC no evidenció progresión de lesiones ni aumento en extensión del compromiso vascular, se decidió retomar quimioterapia con ciclofosfamida asociado a prednisona $(2 \mathrm{mg} / \mathrm{kg} /$ día $)$. 


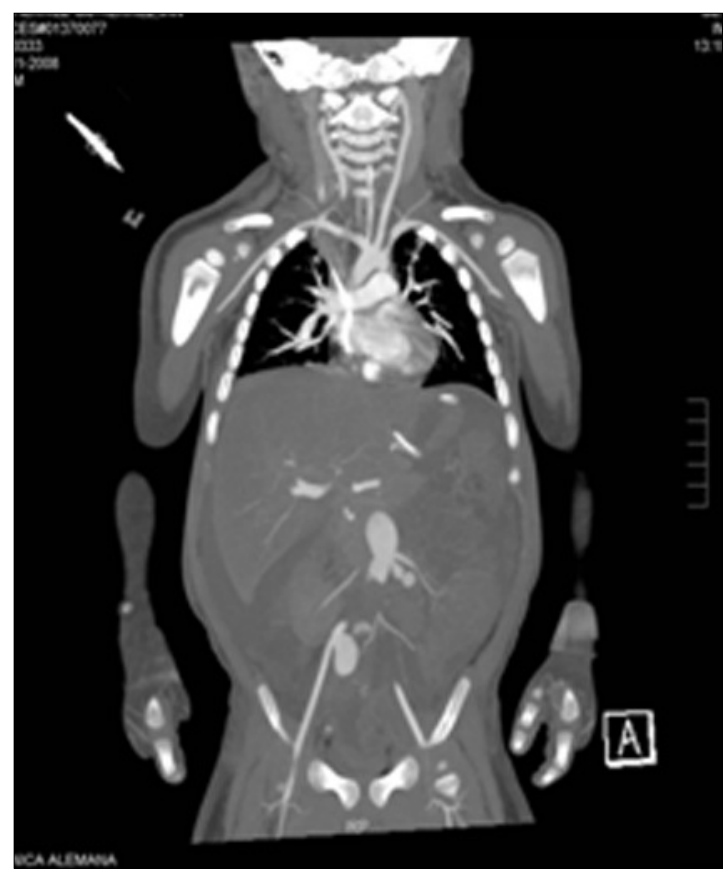

Figura 3. AngioTAC. Se observa trombosis de segmento distal de arteria mesentérica superior aneurismática junto con signos de isquemia de intestino delgado con posible perforación de un asa y colección intraperitoneal.

Dada evolución favorable sin nuevos fenómenos isquémicos ni infecciosos, se realizó 2 meses más tarde (14 meses de edad) reconstitución de tránsito intestinal. Se manejó nutricionalmente con nutrición parenteral (NP) con aumento progresivo de estímulo enteral retirando finalmente NP doce semanas tras reconstitución de tránsito. Tras seis meses de hospitalización fue dado de alta, actualmente se mantiene en controles ambulatorios, evolucionando favorablemente.

\section{Discusión}

La AT resulta ser muy infrecuente en lac$\operatorname{tantes}^{11}$. En la literatura se reportan, en general casos en niños con edad promedio 11,4 años ${ }^{4}$, el caso de menor edad descrito se trata de un lactante chileno, diagnosticado a los 5 meses de edad, pero con una evolución exenta de complicaciones $^{9}$ ). Nuestro paciente a los 6 me- ses ya presentaba criterios de AT de acuerdo a criterios de "Enfermedad activa" (tabla 2).

El paciente debuta con cordón vascular palpable en región pectoral completamente asintomático, el diagnóstico se llevó a cabo gracias a estudio ecográfico y de laboratorio destacando VSG elevada (mantenida sobre $100 \mathrm{mmhr}$ ) sumado a hallazgos clínicos como HTA persistente de díficil manejo, llamativa diferencia de valores de presión arterial entre extremidades inferiores. Si bien no cumplió estrictamente criterios enunciados por la Academia Americana de Reumatología sí se enmarca en criterios de enfermedad activa para pacientes con AT. Esto puede deberse, tal como se representa en la literatura, a que criterios de Academia Americana de Reumatología se manifiestan en etapas más tardías de la enfermedad, mientras que en etapas tempranas prestenóticas cobran más importancia criterios de "Enfermedad Activa" propuestos por Ishikawa ya en el año 1988. Dichos criterios definen como enfermedad activa si aparecen o empeoran de 2 o más rasgos o características de la $\mathrm{AT}$, mientras que una evidente disminución de los síntomas o la mejoría de los hallazgos clínicos indicarían remisión parcial o enfermedad latente. La resolución completa de todos los hallazgos clínicos o su estabilización, en el contexto de lesiones vasculares invariables, sería indicativo de remisión completa ${ }^{5,6}$.

El estudio con imágenes en este tipo de patologías tiene gran valor diagnóstico y en seguimiento, en este caso en particular la angioTAC tuvo un rol fundamental. Al compararla con la angioRNM, mucho más costosa, no presenta mayores beneficios en relación a diagnóstico y seguimiento de este tipo de patologías ${ }^{13}$. Ambos métodos con excelente rendimiento, no invasivos y menos engorrosos que la angiografía, han logrado desplazar a ésta última para este tipo de estudios.

En general el diagnóstico es tardío, muchas veces las primeras manifestaciones clínicas se deben al compromiso isquémico de órganos afectados, series clínicas pediátricas revelan que el tiempo transcurrido hasta el diagnóstico de AT muestra una media de 19 meses $^{5}$. El pronóstico de la AT es incierto, depende del daño, extensión, ubicación de las lesiones. Sin 
embargo la mortalidad en niños supera habitualmente a la de adultos, estimándose en un $33 \%{ }^{4}$.

En cuanto al manejo, y como en la mayoría de las vasculitis, se debe iniciar tratamiento corticoidal con prednisona aumentando progresivamente las dosis hasta alcanzar dosis de mantención en $60 \mathrm{mg} / \mathrm{kg} /$ día para luego comenzar su disminución paulatina ${ }^{12}$, aún cuando hay publicaciones que describen exacerbaciones de la enfermedad al disminuir do$\operatorname{sis}^{3,13}$. En el caso de nuestro paciente, por mala respuesta terapéutica se inició tratamiento de segunda línea con metotrexato combinado con corticoides, ya que se ha demostrado que dicha asociación por tiempos prologados mejora sobrevida $^{12}$, algunos grupos postulan además que este tipo de tratamiento implementado inicialmente puede evitar o disminuir complicaciones.

Sin embargo, en controles con imágenes se objetivó progresión de la enfermedad. No existen reportes de peso en la literatura de tratamiento de tercera línea en lactantes, pero se describe tratamiento con ciclofosmamida, por lo que se inició dicho citotóxico en forma empírica. Estudios recientes proponen tratamiento biasociado ciclofosfamida más a corticoides como segunda línea y mantención con metotrexato, con resultados promisorios por el momento $^{7}$. El uso de anti TNFa (anti Factor de Necrosis Tumoral alfa) ${ }^{8}$ estuvo en discusión, ya que se ha descrito su beneficio en vasculitis refractarias, sin embargo, considerando que aún cuando presentó complicación isquémica no existió progresión en extensión de compromiso vascular, no siendo necesario escalar en tratamiento inmunosupresor.

En cuanto al tratamiento quirúrgico en forma electiva en lactantes, se justificaría sólo en casos con HTA refractaria en los cuales se describe la angioplastía percutánea transluminal para resolver la estenosis secundaria en la arteritis ${ }^{14}$. Siendo este tipo de tratamiento menos efectivo cuando existen múltiples estenosis ${ }^{15}$.

Otro pilar en el manejo es la terapia anticoagulante, que debe iniciarse en forma precoz en la presencia de aneurismas vasculares, en forma ambulatoria para prevenir complicaciones trombóticas. Frente a trombosis arteriales sintomáticas, como en este caso, el tratamiento trombolítico es fundamental para salvar la irrigación del territorio comprometido, sin embargo, en lactantes existe escasa literatura en relación a que agentes utilizar y dosis (urokinasa, estreptoquinasa, activador del plasminógeno tisular), lo que dificulta realizar un tratamiento trombolítico sistémico. La trombolisis local es la terapia de elección ${ }^{16}$ con menos efectos adversos que la sistemica, mejor control y mejores resultados, para su instauración se requiere de un equipo de radiólogos intervensionistas, realidad poco habitual en centros públicos.

Aun cuando este paciente representó complicaciones que ensombrecian su pronóstico gracias al manejo multidisciplinario, involucrando a cirujanos, inmunologos, nutriologos, hematologos, intensivistas, con un un seguimiento estricto de su evolucion clínica, se logro resolver las complicaciones infrecuentes (necrosis intestinal, intestino corto) en el curso de la AT.

\section{Referencias}

1.- Garcia-Consuegra Molina J, et al: Poliarteritis nodosa An Pediatr (Barc) 2005; 62(4): 267-70

2.- Kalangos A, Christenson J, Cikirikcioglu $M$, et al: Long-term outcome alter surgical intervetion and interventional procedures for management of takayasu's arteritis in children. J Thorac Cardiovasc Surg 2006; 132: 656-64.

3.- Kerr G, Hallahan C, Giordano J, et al: Takayasu Arteritis. Ann Intern Med 1994; 120: 919-29.

4.- Fieldston E, Albert D, Finkel T: Takayasu Arteritis in Children and young Indians. Int J Cardiol 2000; 75 (supl 1): S153-S157.

5.- Lacruz Pérez L: Granulomatosis de Wegener y arteritis de Takayasu. An Pediatr (Barc) 2005; 62(4): 271-6.

6.- Ishikawa Kaichiro: Diagnostic Approach and Proposed criteria for de clinical diagnosis of takayasu`s arteriopathy. J Am Coll Cardiol 1988; 12: 964-72.

7.- Ozen S, Duzova A, Bakkaloglu A, et al: Takayasu Arteritis in Children: Preliminary experiencie with cyclosphosphamide induction and corticosteroids folowed by methotrexate. J Pediatr 2007; 150: 72-6.

8.- Sabrina P, Bracaglia C, Campana A, et al: Infliximab Therapy in pediatric Takayasu's arteritis: report of two cases. Rheumatol Int, published online 23 october 
2009.

9.- Saihe A, Carlos Wash F: Arteritis de Takayasu: caso clínico y seguimiento por 23 años. Rev Chil Pediatr [online]. 2007, vol.78, n.3, pp. 284-91.

10.- Baumgartner D, Sailer-Hock M, Baumgartner C, Trieb T, Maurer H, Schrimer M: Reduced aortic elastic properties in a child with Takayasu's arteritis: case report and literature review. Eur J Pediatr 2005; 164: 685-90.

11.- Andrews J, Mason JC: Takayasu's arteritis-recent advances in imaging offer promise. Rheumatology (2007) 46: 6-15.

12.- Ruige JB, Van Geet C, Nevelsteen A: A 16-year survey of Takayasu's Arteritis in a tertiary Belgian centre. Int Angiol 2003; 22: 414-20.
13.- Maksimowicz-McKinnon $K$, Clark T, Hoffman $G$ : Limitations of Therapy and a Guarded Prognosis in an American Cohort of Takayasu Arteritis Patients. Arthritis and Reumatism Vol. 56, No. 3, March 2007, pp 1000-1009.

14.- Sparks SR, Chock A, Seslar S, Bergan JJ, Owens EL: Surgical treatment of Takayasu's Arteritis: case report and review. Ann Vasc Surg 2000; 14: 125-9.

15.- Courtel JV, Soto V, Niaudet P, et al: Percutaneous transluminal angioplasty of renal artery stenosis in children. Pediatr Radiol 1998; 28: 59-63.

16.- Kohler M, Kramann B, Helltern P, et al: Successful treatment of superior mesenteric artery thrombosis with local high-dose urokinase therapy. Klin Wochenschr 1985 Aug 1; 63(15): 722-7. 